\title{
HIGH RESOLUTION X-RAY RECIPROCAL SPACE MAPPING
}

\author{
G. BAUER, J.H. LI
}

Institut für Halbleiterphysik, Johannes Kepler Universität, Linz, 4040 Austria

AND V. HOLY

Faculty of Science, Masaryk University, 61137 Brno, Czech Republic

A survey will be given on recent advances in the investigation of semiconductor epilayers, heterostructures and superlattices using reciprocal space mapping techniques based on triple-axis diffractometry. It is shown that $\mathrm{X}$-ray reciprocal space mapping yields quantitative information on strain, strain relaxation, as well as composition in such structures. These data are obtained from analyses of the isointensity contours of scattered X-ray intensity around reciprocal lattice points. Further analysis of the diffuse scattering yields also information on defect distribution in the epilayers.

PACS numbers: 61.10.-i, 68.55.-a, 61.72.Lk

\section{Introduction}

$\mathrm{X}$-ray reciprocal space mapping has been recently established as a powerful method for strain and structural characterization of epilayers and heterostructures [1-4]. It has the advantage of being non-destructive, probing large sample area, and yielding high precise strain and composition values. A recent development of this method provides also a possibility to extract information on defects, such as statistic distribution of microeffects and dislocation densities in strain relaxed heterostructures, from the diffuse scattering around coherent Bragg reflection peaks in reciprocal space [5-7].

It is the purpose of this paper to demonstrate the usefulness of X-ray reciprocal space mapping for the strain and structure diagnosis of hetero-epitaxial structures. The principle of the method will be described in Sec. 2. In the experimental part, we employ a variety of molecular beam epitaxy (MBE) grown $\mathrm{SiGe} / \mathrm{Si}$ (001) heterosystems as examples. It is demonstrated that by mere inspection of the measured reciprocal lattice maps, the strain status in complex structures becomes obvious, at least qualitatively (Sec. 3). Quantitative information can be obtained by carefully analysing both the coherent as well as the diffuse scattered radiation as apparent in the reciprocal space maps (Sec. 4). 


\section{Reciprocal space mapping}

Figure 1 shows a plane of the reciprocal lattice of a fully relaxed Ge layer on an unstrained Si substrate. The plane of diffraction is defined by the incident and the diffracted X-ray beams which are chosen to lie in the plane determined by the two crystallographical [001] and [110] directions. The two small half circles (Laue zones, radius: $1 / \lambda, \lambda$ being the $\mathrm{X}$-ray wavelength) and the large one (radius: $2 / \lambda$ ) indicate the limits of accessibility for diffractometric measurements in the Bragg (reflection) geometry. The Ewald sphere construction is shown for the Si (115) high incidence reflection. The Bragg condition is fulfilled when the diffraction vector $h$ equals a reciprocal lattice vector. The diffractometer angle $\omega$ is defined as that between the incident $\left(k_{\text {inc }}\right)$ beam and the sample surface, and the angle $2 \Theta$ is that between the incident and the diffracted $\left(k_{\text {diff }}\right)$ beams. Three possible scan directions, i.e., the $\omega / 2 \Theta$ ("a"), the $\omega$ (" $b$ "), and the $2 \Theta(" c$ ") scan directions, are depicted in Fig. 1. An ideal diffractometer (neither axial nor vertical divergence, and assuming a perfectly monochromatic $\mathrm{X}$-ray beam) collects intensity transversal in reciprocal space (arrow " $b$ ") along a circle with centre (000), the origin of the reciprocal lattice, if the sample is rotated, i.e., if $\omega$ is changed ( $\omega$-scan direction). If the detector is moved with the sample position being fixed (i.e., the angle $2 \Theta$

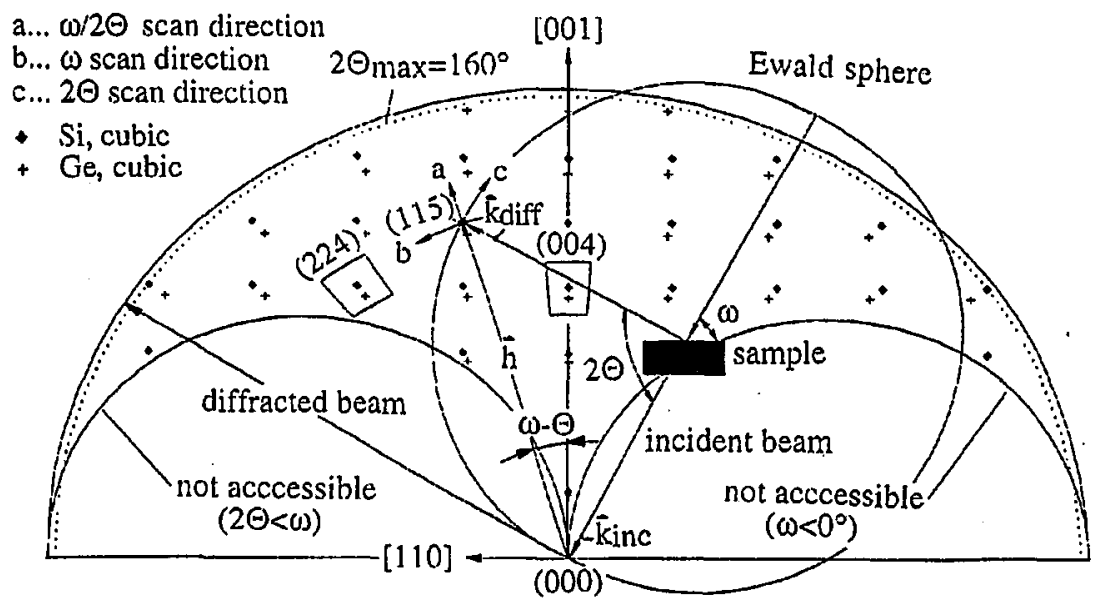

Fig. 1. Schematic representation of the reciprocal lattice points of fully relaxed [001] oriented Si (full diamonds $\diamond$ ) and Ge $(+)$ accessible by X-ray diffraction in the plane of diffraction in reciprocal space with [110] azimuth and $\mathrm{Cu} K_{\alpha_{1}}$ radiation. Limits for the accessibility are the maximal diffractometer angle $2 \Theta=160^{\circ}$ and the Lave zones $\left(\omega<0^{\circ}\right.$ and $2 \Theta>\omega$ ). The Ewald sphere construction shows the incident and the diffracted wave vectors $k_{\text {inc }}$ and $k_{\text {diff }}$, and the three possible scan directions in reciprocal space (" $a$ ", " $b$ " and " $c$ "). The regions where reciprocal space maps have been measured by combining the directions " $a$ " and " $b$ " around (224) and (004) reciprocal lattice points are indicated schematically. (000): origin of reciprocal lattice at $2 \Theta=0, \omega=$ arbitrary. 
changes), intensity will be collected along the Ewald sphere (arrow "c", $2 \Theta$-scan direction). Finally, if the detector is rotated $t$ wice as fast around the diffractometer axis as the sample, the scan direction in reciprocal space is radial from (000) (arrow " $a$ ", $\omega / 2 \Theta$-scan direction). Reciprocal space mapping is usually done by making a series of $\omega / 2 \Theta$ scans at different $\omega$ settings.

For the strain analysis, we map the regions marked in Fig. 1. Qualitatively, strain and structural information can be obtained directly from the recorded reciprocal space maps. The principle which relates the relative position of the reciprocal lattice point (RELP) of an epilayer with respect to that of the substrate in reciprocal space is schematically shown in Fig. 2. The Si-substrate is assumed to be unstrained, and from the relative position of substrate and layer RELP maxima in both symmetrical and asymmetrical maps (e.g. (004) and (224) maps, respectively) one directly obtains the in-plane lattice constant $\left(a_{\mathrm{p}}\right)$, and the lattice constant in growth direction $\left(a_{n}\right)$ of the layer without assumptions on elastic constants as described in Fig. 2. The distance between a layer RELP maximum and the origin $(000)$ along the direction [110] is inversely proportional to the in-plane lattice constant. If a Ge layer, which has a larger bulk cubic lattice constant than that of the $\mathrm{Si}$ substrate, is grown pseudomorphically, i.e., tetragonally distorted ( $\mathrm{Ge}^{\text {pseu }}$ in Fig. 2), it has the same in-plane lattice constant as the substrate. Its RELP maximum lies underneath that of the Si substrate along the growth direction [001] in any of the asymmetrical maps. In the other extreme, if the Ge-layer is fully relaxed and cubic ( $\mathrm{Ge}^{\text {rel }}$ in Fig. 2), asymmetrical lattice planes of layer and substrate are parallel to each other, and the layer RELP maximum lies underneath that of the substrate along the direction $[h k l]$ through the $(h k l)$ substrate RELP maximum as shown in Fig. 2. Obviously; a partially relaxed Ge layer gives rise to a RELP located in between these two extreme positions of substrate and layer RELP's described above (bold arrows in Fig. 2).

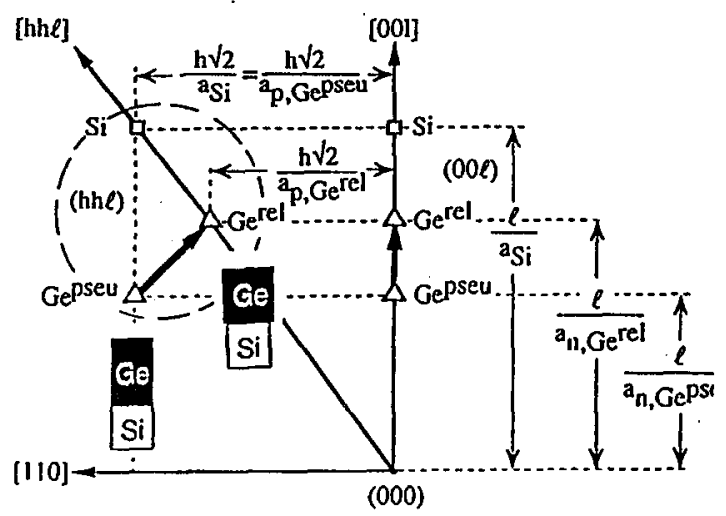

Fig. 2. Schematic representation of correlation between distances in reciprocal space and lattice constants in $\left(a_{n}\right)$ and perpendicular $\left(a_{p}\right)$ to [001] growth direction for the example of a pseudomorphic ( $\left.\mathrm{Ge}^{\text {pseu }}\right)$ and fully relaxed $\left(\mathrm{Ge}^{\text {rel }}\right)$ Ge layer on Si. The bold arrows describe the path of the layer RELP in reciprocal space during relaxation. 
For quantitative analyses, some critical parameters have to be defined. In the asymmetrical ( $h h l)$ map (Fig. 2) the angle $\alpha$ between the bold arrow, which describes the path of the layer RELP during relaxation, and the [001] growth direction is given by $[3,8]$ :

$$
\alpha=\arctan \left[\frac{\tan (\omega-\theta)}{\frac{2 C_{12}}{C_{11}}}\right],
$$

where the denominator includes the elastic stiffnesses of the layer and the angle $(\omega-\Theta)$ depends on the particular asymmetrical reflection [see Fig. 1, (115) reflection].

We denote the angles $(\omega-\theta)$ and $\Theta$ (see Fig. 1) as $\Omega$ and $\Lambda$ for simplicity. These two angles fully describe the position of a RELP in reciprocal space. If two smaller maps around the substrate and the layer RELP's are measured separately instead of one large map covering both RELP's, the positions of the centres of these maps are denoted by $\omega_{S}$ and $\Theta_{S}$ for the substrate- and $\omega_{L}$ and $\theta_{L}$ for the layer-map. The substrate is used as an internal standard, and the values $\Omega_{\mathrm{S}}$ and $\Lambda_{\mathrm{S}}$ for the unstrained substrate are known from simple geometrical considerations and from Bragg's law. Then the angles $\Omega_{\mathrm{L}}$ and $\Lambda_{\mathrm{L}}$ defining the relative position of the layer RELP with respect to the substrate RELP are

$$
\begin{aligned}
& \Omega_{\mathrm{L}}=\Omega_{\mathrm{S}}+\Delta \Omega_{\mathrm{S}}-\Delta \Omega_{\mathrm{S}}+\omega_{\mathrm{L}}-\omega_{\mathrm{S}}-\Theta_{\mathrm{L}}+\Theta_{\mathrm{S}}-\Omega_{\mathrm{tilt}}, \\
& \Lambda_{\mathrm{L}}=\Lambda_{\mathrm{S}}+\Delta \Lambda_{\mathrm{L}}-\Delta \Lambda_{\mathrm{S}},
\end{aligned}
$$

where $\Delta \Omega_{\mathrm{L}}, \Delta \Omega_{\mathrm{S}}$ and $\Delta \Lambda_{\mathrm{L}}, \Delta \Lambda_{\mathrm{S}}$ describe the angular distances of layer and substrate RELP's from the centres of the measured asymmetrical maps in the $\omega$ and $\omega / 2 \Theta$-scan directions. For the strain analysis the tilt angle $\Omega_{\text {tilt }}$ has to be taken into consideration [8-10]. The symmetrical and asymmetrical layer RELP's have to be rotated in reciprocal space by the same angle $\Omega_{\text {tilt }}$ along the $\omega$-scan direction, so that the symmetrical layer RELP lies underneath that one of the substrate along the growth direction.

From the angular positions of the layer peaks in the asymmetrical map, the reciprocal lattice vector components $b_{z}$ and $b_{x}$ of the layer along and perpendicular to the growth direction are directly obtained [3]:

$$
b_{z}=2 K \sin \left(\Lambda_{\mathrm{L}}\right) \cos \left(\Omega_{\mathrm{L}}\right), \quad \text { and } \quad b_{x}=2 K \sin \left(\Lambda_{\mathrm{L}}\right) \sin \left(\Omega_{\mathrm{L}}\right),
$$

where $K=1 / \lambda$ is the absolute value of the wave vector in vacuum. For [001] oriented growth and the asymmetrical (224) reflection the reciprocal lattice vector components depend on the in-plane lattice constant $\left(a_{\mathrm{p}}\right)$ and that in growth direction $\left(a_{\mathrm{n}}\right)$ of the layer

$$
b_{z}=4 / a_{\mathrm{n}}, \quad \text { and } \quad b_{x}=2 \sqrt{2} / a_{\mathrm{p}} .
$$

It is obvious then that the use of the (224) reflection offers about a two times higher accuracy than the commonly used (115) reflection for the analysis of the in-plane strains, because the angle $\Omega=\omega-\Theta$ is about twice as large for the (224) reflection.

The situation becomes more complex, if the deviation of the composition dependence of the lattice constant from Vegard's law for $\mathrm{Si}_{1-x} \mathrm{Ge}_{x}$ alloys [11] is 
taken into account as has been shown in Ref. [3]. Any deviation from Vegard's law causes a separation between the RELP's from a strained layer superlattice (SLS) and an alloy layer with the same mean composition and furthermore their relaxation lines in the reciprocal space maps are not parallel to each other [3].

In the case of graded SiGe layers [12-19], the strain analysis is complicated since the RELP positions are different for different sublayers and the averaged strains depend on the position $z$ along growth direction. In order to determine the averaged strains in graded layers, both (004) and (224) reciprocal space maps (RSM's) have to be analyzed. The principle of this analysis is shown in Fig. 3. In a first step, the diffuse intensity maximum in the (004) RSM is decomposed

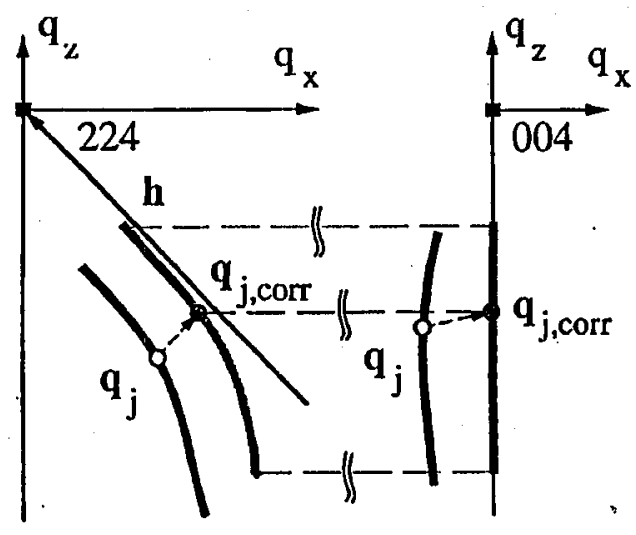

Fig. 3. Sketch of distribution of the diffuse X-ray scattering from a graded SiGe buffer in reciprocal plane. The thick black and grey curves denote the shape of the intensity maxima before and after the tilt correction, respectively. The circle expresses the maximum of the diffuse X-ray scattering originating in the $j$-th sublayer of the graded buffer, the substrate RELP is denoted by the full square.

into a set of $N$ thin strips parallel to the $q_{z}$-axis. The diffusely scattered intensity contributing to the $j$-th strip mainly originates from the $j$-th sublayer of the graded layer. In each strip, we find the position $q_{x j}^{004}$ of the intensity maximum and this value determines the tilt $\Omega_{j}$ of the $j$-th sublayer and is independent of its degree of relaxation. In the second step, the positions of the maxima of the diffuse intensity distribution are corrected for the tilt angles $\Omega_{j}$, and thus the corrected maxima in the (004) and (224) RSM's having the same $q_{z}$-coordinate correspond to the same sublayer and then the procedure as described above is used in order to determine the in-plane and normal lattice constants of the $j$-th sublayer. With the aid of the elastic constants, the relaxed lattice constant is obtained and thus the position $z^{j}$ with the aid of a known Ge profile [18].

\section{Multilayer structures}

In this section, the power of reciprocal space mapping on the strain and structural characterization of complex multilayer systems is shown. Only a quali- 
tative description will be given in this section. Examples of quantitative analyses of reciprocal space maps will be given in Sec. 4 .

\subsection{Pseudomorphic layers}

As an example, a multiquantum well (MQW) structure is investigated here. The structure consists of 10 periods of quantum well with 4.3 monolayers (ML's) of $\mathrm{Ge}$, followed by $21.5 \mathrm{ML}$ 's of Si, followed by $4.3 \mathrm{ML}$ 's of $\mathrm{Ge}, 8.8 \mathrm{~nm}$ of $\mathrm{Si}_{0.84} \mathrm{Ge}_{0.16}$, and $45.1 \mathrm{~nm}$ of $\mathrm{Si}$ (data follow from the dynamical simulation of X-ray rocking curve). On top of this structure a $\mathrm{Si}_{0.963} \mathrm{Ge}_{0.037}$ waveguide was grown terminated with a Si-cap layer $(10 \mathrm{~nm})$. For a comparison of conventional double crystal diffraction (DCD) data and reciprocal space mapping on pseudomorphic structures, we show both data in Figs. $4 \mathrm{a}$ and $4 \mathrm{~b}$, respectively. The overall MQW-intensity is modulated by the thin Si and Ge layers. In the reciprocal space map, which shows just a small portion of the DCD diffraction structure, the $\mathrm{Si}$ substrate and the satellite peaks from the MQW structure are shown for the symmetric (004) and the asymmetric (224) maps. The FWHM's of all contours of constant intensity extrema along the $\omega$-direction correspond exactly to the experimental resolution of $12 \mathrm{arcsec}$ and all corresponding intensity extrema have the same in-plane lattice constant. Thus this sequence of layers is entirely pseudomorphic.

Thus reciprocal space mapping yields in this case information whether a sequence of layers is indeed grown pseudomorphically on the substrate or not. The rocking curve analysis is needed in addition to obtain the proper layer thicknesses from dynamical simulations.

\subsection{Relaxed Si/Ge structures}

An example of a quite elaborate strain and composition analysis of $\mathrm{Si} / \mathrm{SiGe}$ short period superlattice sample grown on a graded buffer using a growth concept which was suggested by Schäfler et al. [20] is shown in Figs. 5a and 5b. The sample consists nominally of a linearly graded SiGe buffer on which a constant alloy buffer was deposited with a $\mathrm{Ge}$ content lower than that of the top layer of the graded buffer. Furthermore, a nominally 100 period $\mathrm{Si} / \mathrm{Si}_{0.5} \mathrm{Ge}_{0.5}$ superlattice (SL) was deposited on top of this constant buffer. From the inset of Fig. 5a which shows the results of the secondary ion mass spectroscopy (SIMS) analysis of this sample, it is clear that the average Ge-content in the SL region of the sample changes abruptly, i.e. there are actually two SL's grown with different mean Ge-content. In Fig. 6a the DCD rocking curve for a (004) Bragg diffraction is shown while in Fig. 6b the reciprocal space maps around the (004) and (224) lattice points are shown. From the DCD data alone it is clear that a simulation without further knowledge on the strain status of the layers is quite difficult or even impossible. The strain and composition analysis based on the data of the reciprocal space maps reveals that the buffer $B 2$ grows pseudomorphically on the top layers of $B 1$, and that in addition the two SL's denoted by SL and by $\mathrm{SL}^{\prime}$ grow pseudomorphically on this buffer $B 2$. On top of the entire structure there is a SiGe cap layer. From the analysis it follows that the Ge content in the graded buffer $B 1$ increases linearly 

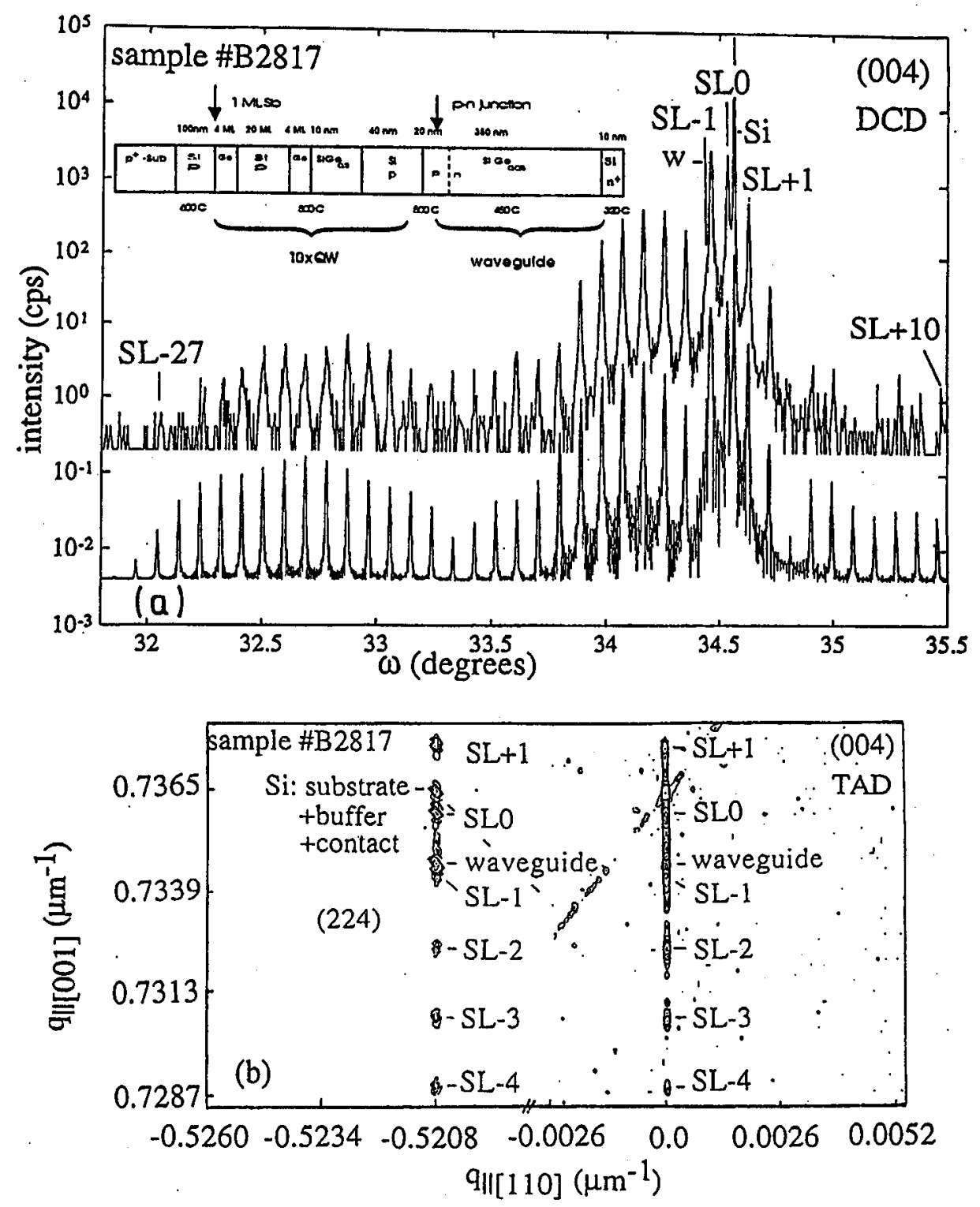

Fig. 4. (a) Upper trace: (004) DCD Bragg reflection curve from a pseudomorphic short period $\mathrm{Si} / \mathrm{Ge}$ structure grown on (001) Si substrate with a SiGe waveguide structure on top (structural details: insert). Lower trace: dynamical simulation. (b) Reciprocal space maps of sample \#B2817 around the (004) and (224) RELP's showing main superlattice and satellite reflection from the MQW structure, and RELP from the SiGe waveguide, demonstrating that the entire structure is pseudomorphic. FWHM along $\omega$-direction: 12 arcsec. 

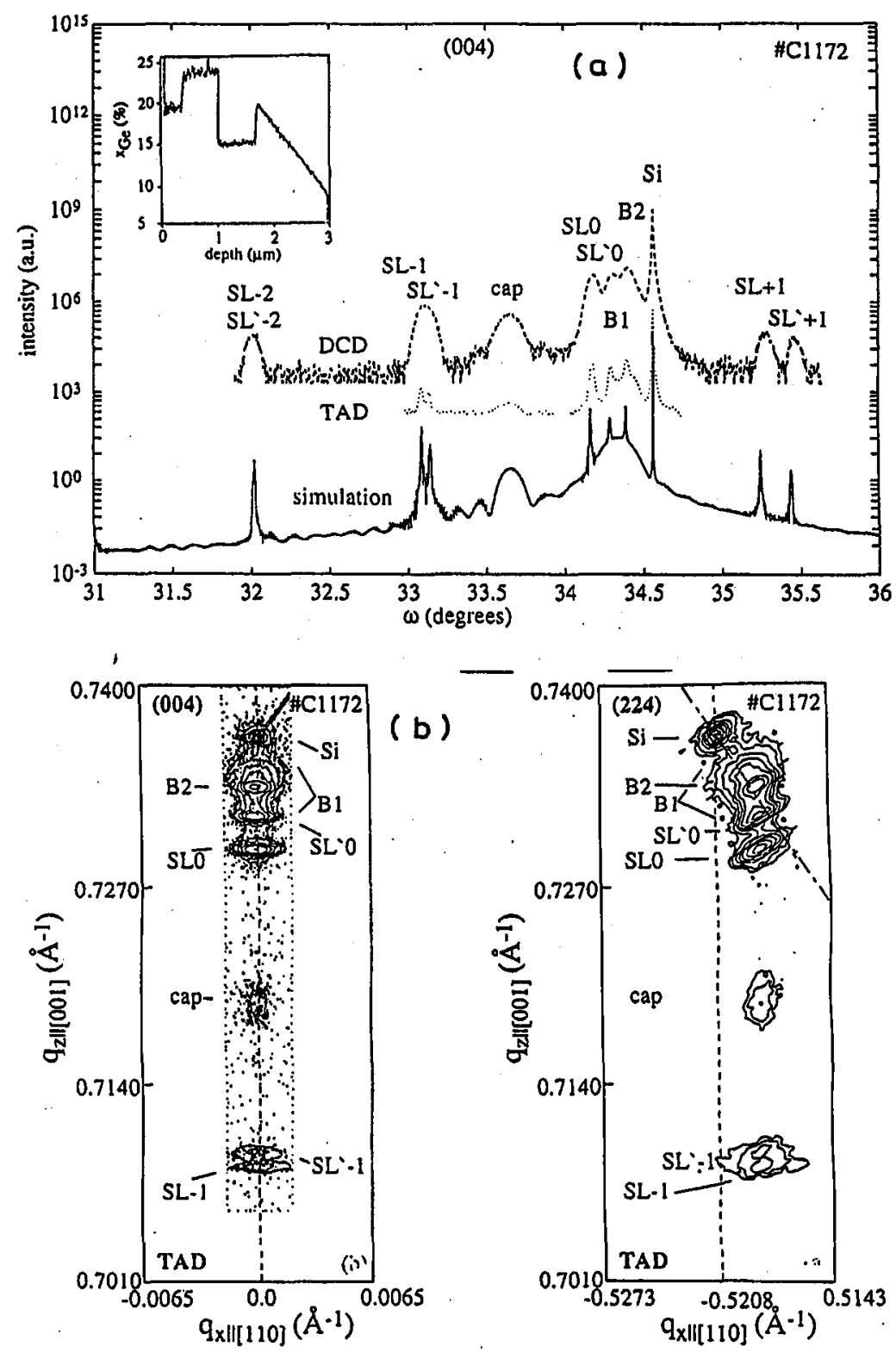

Fig. 5. (a) DCD, TAD and dynamical simulation of a Si/SiGe short period SLS grown on a linearly graded SiGe buffer, followed by a buffer layer with constant Ge content. Inset: results of a SIMS analysis of the change of Ge-content with depth from the surface. (b) Reciprocal space maps of this structure around (004) and (224) showing the relaxation of the linearly graded buffer $B 1$ and the strain status of $B 2$, and of the two $\mathrm{Si} / \mathrm{SiGe}$ superlattices $\mathrm{SL}$ and $\mathrm{SL}^{\prime}$, respectively. 

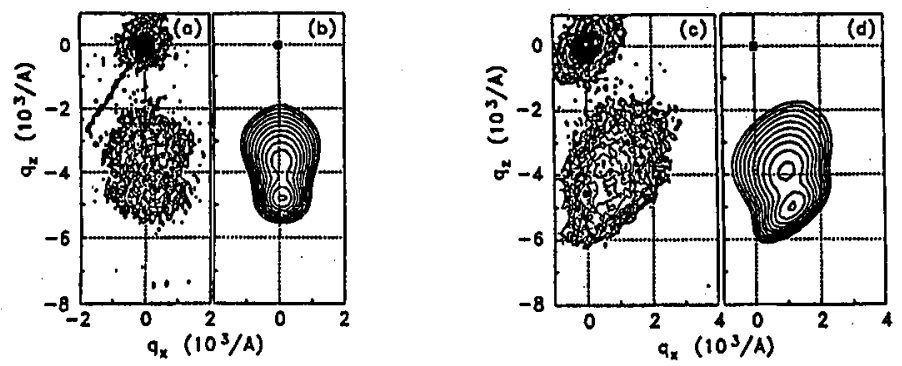

Fig. 6. (a) Measured and (b) calculated (004) reciprocal space maps of sample C1444. (c) Measured and (d) calculated (224) reciprocal space maps of sample C1444.

from 9 to $20 \%$, with a thickness of $1.3 \mu \mathrm{m}$, the buffer $B 2$ is $650 \mathrm{~nm}$ thick with a Ge content of $14.5 \%$ and has an in-plane lattice constant of $5.4575 \AA$. The in-plane lattice constants of the two SL's are almost the same as the one of $B 2$ $(5.4570 \AA)$, but the Ge content in the SiGe layers of SL is $48 \%$ whereas in the second $\left(\mathrm{SL}^{\prime}\right)$ superlattice it is just $40 \%$. In accordance with the SL periods of $46.4 \AA$ and $46.6 \AA$ determined from the angular distances of the SL0 and SL-1, and SL $0^{\prime}$ and SL-1' RELP's, it follows that the layer thicknesses are $24.3 \AA$ for the $\mathrm{Si}$ and $22.1 \AA$ for the $\mathrm{Si}_{0.6} \mathrm{Ge}_{0.4}$ layers in $\mathrm{SL}^{\prime}$ (80 periods), and $24.3 \AA \mathrm{Si}$ and $22.3 \AA \mathrm{Si}_{0.52} \mathrm{Ge}_{0.48}$ in SL (20 periods). The $40 \mathrm{~nm}$ thick cap layer has a Ge content of $40 \%$. The average Ge contents in the two superlattices of $23 \%$ and $19 \%$ for $\mathrm{SL}^{\prime}$ and SL, respectively, are in reasonable agreement with the results of the SIMS measurement. In Fig. 6a the two experimental traces correspond to the DCD and the triple-axis diffractometry (TAD) measurements, and it is obvious that only in the TAD data the resolution is sufficiently high to allow for a proper comparison with the dynamical calculations (also shown in Fig. 6a, for an in-depth discussion see Ref. [21]), which is based on the strain data evaluated from the reciprocal space maps. The analysis of a $\mathrm{Si} / \mathrm{SiGe}$ sample like $\mathrm{C} 1172$ provides a crucial test for the usefulness of reciprocal space mapping.

\section{Graded buffer layers}

Whenever a two-dimensional electron gas is realized with $\mathrm{Si} / \mathrm{SiGe}$ structures, first a partially or fully relaxed SiGe alloy buffer has to be grown on the Si substrate to provide the proper biaxial tensile strain status in the subsequent $\mathrm{Si}$ quantum well layers $[12,15,20]$. Consequently, the composition and the strain status of the $\mathrm{SiGe}$ alloy buffer is particularly crucial for the resulting band alignment at the $\mathrm{Si} / \mathrm{Ge} / \mathrm{Si}$ interfaces. Reciprocal space mapping is quite suitable for providing this structural information. As an example, graded buffers with linearly increasing $\mathrm{Ge}$ contents are studied here. The two samples (C1444 and C1446) were grown by MBE on Si (001) substrates with a Ge gradient of about $15 \% / \mu \mathrm{m}$ and thicknesses of $0.4 \mu \mathrm{m}$ (C1444) and $1 \mu \mathrm{m}$ (C1446), respectively. The starting Ge content for both samples is about $7 \%$.

The measured (004) and (224) reciprocal space maps for samples C1444 and C1446 are shown in Figs. 6a, c and 7a, c respectively. Based on the method described in Fig. 3, we determine first the mean in-depth strain distribution according 

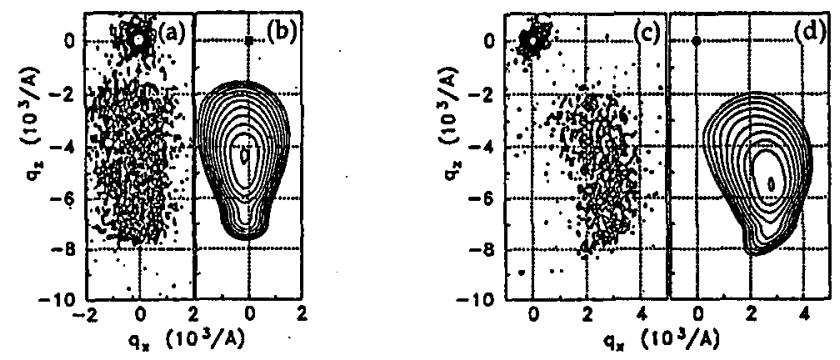

Fig. 7. (a) Measured and (b) calculated (004) reciprocal space maps of sample C1446. (c) Measured and (d) calculated (224) reciprocal space maps of sample C1446.

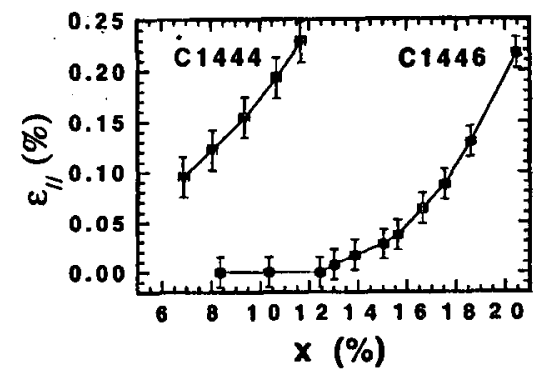

Fig. 8. Strain profile as a function of Ge content of samples C1444 and C1446. Both strain and composition data were extracted from X-ray reciprocal space maps.

to the coherent diffraction, which determines the position of the reciprocal lattice point of the epilayer in reciprocal space. The resulting strain data are shown in Fig. 8 versus Ge content. It is clearly seen that for sample C1444 only the lowermost layer close to the substrate surface is partially strain relaxed, whereas the rest part remains fully strained on this partially relaxed layer. Sample C1446 has a larger thickness, therefore, the lower part close to the substrate is completely relaxed, whereas the topmost part again remains pseudomorphic. Furthermore, it is found that a partially relaxed interlayer exists in between of the completely relaxed lower layer and the fully strained top layer, which is believed to be caused by kinetic limitation of dislocation nucleation and glide processes due to the finite growth temperature employed in our experiments [18]. It is worth noting that even if the final Ge content of both samples differs significantly, the residual strains near the film surface are quite the same. This means that the strain conditions will remain constant after the on-set of dislocation nucleation as long as the gradient and growth temperature are kept constant.

Now, let us consider the diffuse scattering. In each of the measured reciprocal space maps, the reciprocal lattice point of the epilayer is broadened along the direction perpendicular to the diffraction vectors due to the diffuse scattering from defects. In our case, the dominant defects are misfit dislocations. We have shown recently that misfit dislocations cause randomly distributed strain fields in the 
epilayer [22]. These random strains lead to the diffuse scattcred intensity around the coherent diffraction peaks. Therefore, by calculating the scattering from the random strains distributed in the SiGe epilayers using a kinematic theory based on considerations previously suggested by Krivoglaz [23], we reproduce theoretically the intensity distribution in the reciprocal space maps. This analysis provides furthermore information on the in-depth distribution of dislocations [18, 22].

The calculated reciprocal space maps for both samples are plotted besides their experimental counterparts shown in Figs. 6 and 7, respectively (Figs. 6b, d and Figs. 7b, d). As can be seen, the fit is quite good. The dislocation profile used to simulate Figs. $6 \mathrm{~b}$ and $\mathrm{d}$ (sample $\mathrm{C} 1444$ ) is a planar misfit dislocation distribution at the epilayer to substrate interface, whereas the remaining part of the epilayer is free of misfit dislocations, because only the interface layer of this sample is partially relaxed. Its density is determined by the $7 \%$ starting Ge content and the degree of relaxation of the interface layer. The dislocation densities used to calculate Figs. 7b and d (sample C1446) are plotted in Fig. 9 versus the distance to the substrate surface. It is obvious that in the completely relaxed part, dislocations are homogeneous. Their density decreases in the partially relaxed region, and reaches

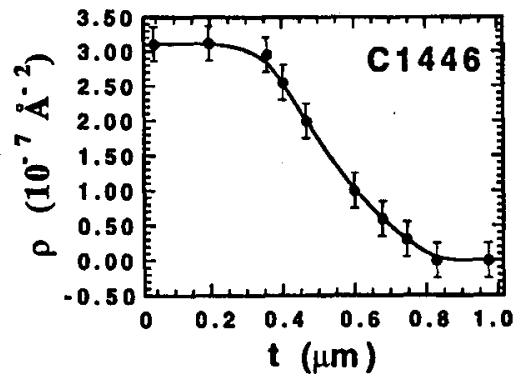

Fig. 9. In-depth distribution of misfit dislocation density of sample C1446 used for the calculation of Fig. $7 \mathrm{~b}$.

zero at the fully strained top layer. We observed also that the intensity distribution in the calculated maps for C1446 is little bit narrower than the measured one in the fully strained region. This is due to the fact that the threading dislocation segments are not considered in our calculation, which, however, actually exist in the samples.

\section{Conclusions}

The potential of reciprocal space mapping based on triple-axis diffractometry [21] for the analysis of the strain status in pseudomorphic, partially and fully relaxed $\mathrm{Si} / \mathrm{SiGe}$ structures has been demonstrated by several groups $[1-4,9,13,24]$. This method is also suitable for a determination of the varying degree of relaxation and of the Ge content in graded SiGe buffer layers and thus yields the necessary information for refined theoretical models on the strain relaxation in complex multilayer structures. Furthermore from the analysis of the diffusely scattered 
radiation also information on the defect structure in $\mathrm{Si} / \mathrm{SiGe}$ heterostructures and superlattices can be derived [6, 24-26].

\section{Acknowledgments}

The authors are grateful to E. Koppensteiner, E. Kasper, F. Schäfler, H. Kibbel, H. Presting, J. Nützel and G. Abstreiter for providing samples, for cooperation and helpful discussions. Work supported by Fonds zur Förderung der wissenschaftlichen Forschung, \# 9119 PHY and 10083 PHY and ESPRIT basic research project No. 7128 , and GME, Vienna.

\section{References}

[1] P.F. Fewster, Semicond. Sci. Technol. 8, 1915 (1993), Appl. Phys. A 58, 121 (1994).

[2] B.K. Tanner, in: Analysis of Microelectronic Materials and Devices, Eds. M. Grasserbauer, H.W. Werner, Wiley, Vienna 1991, p. 609.

[3] E. Koppensteiner, G. Bauer, H. Kibbel, E. Kasper, J. Appl. Phys. 76, 3489 (1994).

[4] C.R. Wie, Mater. Sci. Eng. R 13, 1 (1994).

[5] V. Holy, J. Kubena, K. Ploog, Phys. Status Solidi B 162, 347 (1990).

[6] V. Holy, J. Kubena, E. Abramof, A. Pesek, E. Koppensteiner, J. Phys. D, Appl. Phys. 26, A146 (1993).

[7] P.F. Fewster, N.L. Andrew, J. Appl. Crystallogr. 26, 812 (1993).

[8] P. van der Sluis, Philips J. Res. 47, 203 (1993).

[9] P.M. Mooney, F.K. LeGoues, J. Tersoff, J.O. Chu, J. Appl. Phys. 75, 3968 (1994); P.M. Mooney, F.K. LeGoues, J.L. Jordan-Sweet, Appl. Phys. Lett. 65, 2845 (1994).

[10] A. Segmüller, M. Murakami, in: Treatise on Materials Science and Technology, Vol. 27, Eds. K.N. Tu, R. Rosenberg, Academic Press, New York 1988, p. 143.

[11] J.P. Dismukes, L. Ekstrom, R.J. Paff, J. Phys. Chem. 68, 3021 (1964).

[12] E. Kasper, F. Schäffler, in: Semiconductors and Semimetals, Vol. 33, Ed. T.P. Pearsall, Academic Press, Boston 1991, p. 223.

[13] E. Koppensteiner, P. Hamberger, G. Bauer, A. Pesek, H. Kibbel, H. Presting, E. Kasper, Appl. Phys. Lett. 62, 1783 (1993).

[14] W. Jäger, D. Stenkamp, P. Ehrhardt, K. Leifer, W. Sybertz, H. Kibbel, H. Presting, E. Kasper, Thin Solid Films 222, 221 (1992).

[15] E.A. Fitzgerald, Y.-H. Xie, D. Monroe, P.J. Silverman, J.M. Kuo, A.R. Kortan, F.A. Thiel, B.E. Weir, J. Vac. Sci. Technol. B 10, 1807 (1992); G.P. Watson, E.A. Fitzgerald, Y.-H. Xie, D. Monroe, J. Appl. Phys. 75, 263 (1994).

[16] P.M. Mooney, F.K. LeGoues, I.O. Chu, S.F. Nelson, Appl. Phys. Lett. 62, 3464 (1993).

[17] P.M. Mooney, F.H. Dacol, J.C. Tsang, J.O. Chu, Appl. Phys. Lett.62, 2069 (1993).

[18] J.H. Li, V. Holy, G. Bauer, M. Hohnisch, H.-J. Herzog, F. Schäffler, Acta Phys. Pol. $A$ 89, (1996).

[19] J. Tersoff, Appl. Phys. Lett. 62, 693 (1993).

[20] F. Schäffler, D. Többen, H.J. Herzog, G. Abstreiter, B. Holländer, Semicond. Sci. Technol. 7, 260 (1992).

[21] P. Zaumseil, U. Winter, Phys. Status Solidi A 70, 497 (1982). 
[22] V. Holy, J.H. Li, G. Bauer, F. Schäffler, H.J. Herzog, J. Appl. Phys. 78, 5013 (1995). [23] M.A. Krivoglaz, Theory of X-Ray and Thermal Neutron Diffraction by Real Crystals, Plenum, New York 1969.

[24] E. Koppensteiner, P. Hamberger, G. Bauer, V. Holy, E. Kasper, Appl. Phys. Lett. 64, $172(1994)$.

[25] E. Koppensteiner, G. Bauer, V. Holy, E. Kasper, Jpn. J. Appl. Phys. 33, 440 (1994).

[26] G. Bai, M.-A. Nicolet, C.H. Chern, K.L. Wang, J. Appl. Phys. 75, 4475 (1994). 